\title{
Verfassungsbeschwerde gegen das Gesetz zur Neuregelung von Luftsicherheitsaufgaben
}

- Bemühungen zur Abwehr des finalen Rettungstotschlags -

Die sechs Beschwerdeführer, die von Burkhard Hirsch vertreten werden, rügen mit ihm wegen zentraler Vorschriften des Gesetzes zur Neuregelung von Luftsicherheitsaufgaben ${ }^{1}$ die Verletzung ihrer Grundrechte aus Art. 1 Abs. 1, 2 und 3, Art. 2 Abs. 2, Art. 19 Abs. 2 GG. $^{2}$ Der Verf. referiert im Folgenden die Verfassungsbeschwerde mit Sachverhalt und Begründung in leicht gekürzter und redaktionell bearbeiteter Form.

\section{Zum Sachverhalt der Verfassungsbeschwerde}

\section{Ermächtigung des Staates zu vorsätzlicher Tötung ziviler Opfer}

Durch das angefochtene Gesetz soll dem Staat erlaubt werden, vorsätzlich Menschen zu töten, die nicht Täter, sondern Opfer eines Verbrechens geworden sind. Der Verteidigungsminister der Bundesrepublik Deutschland soll das Recht bekommen, den Abschuß eines Luftfahrzeugs, also auch einer vollbesetzten Passagiermaschine, anzuordnen, »wenn den Umständen nach davon auszugehen ist, daß das Luftfahrzeug gegen das Leben von Menschen eingesetzt werden soll« und »die unmittelbare Einwirkung mit Waffengewalt« das einzige Mittel zur Abwehr der gegenwärtigen Gefahr sei. Diese Formulierung verschleiert den gewollten Sachverhalt: Die Insassen des Flugzeugs kommen im Text des Gesetzes schon nicht mehr vor, mögen es die Mitglieder der Besatzung, die Passagiere sein oder die mutmaßlichen Täter einer Flugzeugentführung, die das Flugzeug »den Umständen nach « gegen das Leben von Menschen einsetzen wollen. Sie alle, Täter und Opfer, werden gemeinsam abgeschossen und getötet. Es kommen auch die Menschen nicht vor, die in der dichtbesiedelten Bundesrepublik möglicherweise am Boden von der abgeschossenen Maschine in den Tod gerissen werden.

Es ist die Einführung des finalen Rettungstotschlags. Die entführten Passagiere und die anderen an der Entführung nicht beteiligten Opfer des herbeigeführten Absturzes sind nur noch Objekte staatlichen Handelns, bedauerliche Kollateralschäden. Die Passagiere sind sozusagen schon tot. Sie sind die Späne, die eben fallen, wenn man hobeln muß. Der verbleibende Rest ihres Lebens zählt nicht mehr. Ihr Grundrecht auf Leben verringert sich anscheinend mit der ihnen - nach Meinung des Verteidigungsministers oder seines Vertreters im Amt - noch verbleibenden Lebenszeit. Sie sollen zugunsten des Lebens anderer Menschen, die mutmaßlich von einem Angriff bedroht sind, durch einen militärischen Einsatz der Bundeswehr bewußt geopfert werden. Und

1 Gesetz vom 11. Jan. 2005 (BGBl. 2005 I, S. 78 ff.).

2 Nach den Vorträgen der Beschwerdeführer sind die $\S 13,14$ und 15 des Luftsicherheitsgesetzes (LuftSiG) mit dem Grundgesetz nicht vereinbar und daher nichtig. 
die am Boden getroffenen Absturzopfer haben eben einfach Pech gehabt, sie waren ja gar nicht gemeint.

Es mag hier dahingestellt bleiben, ob die Verfasser des Gesetzes die merkwürdige Formulierung des $\S 14$ Abs. 3 LuftSiG mit Rücksicht auf Art. 2 Abs. 2 a EMRK oder aus Gründen der politischen Optik gewählt haben. Sie ist jedenfalls die Folge einer der Bedeutung des Sachverhalts nicht angemessenen politischen und parlamentarischen Auseinandersetzung zwischen Mehrheit und Opposition über Zulässigkeit und Umfang eines Einsatzes der Bundeswehr im Inland gewesen. Die Bundesregierung glaubte schließlich versuchen zu sollen, den kriegsmäßigen Einsatz der Bundeswehr mit militärischen Mitteln im Inland als polizeirechtliche Amtshilfe für die Bundesländer zu konstruieren, um die Regelung des Art. 84a GG zu umgehen.

a) Hintergrund der Diskussion: Irrflug in Frankfurt am Main

Nach dem Attentat vom 11. September 2001 in den Vereinigten Staaten auf das World Trade Center und das Pentagon begann dort eine öffentliche Diskussion darüber, ob es nicht möglich gewesen wäre, die entführten Passagierflugzeuge im »war on terror« abschießen zu lassen. Daß es den Passagieren der einen damals entführten Maschine fast gelungen wäre, sich selbst zu befreien, spielte dabei keine erkennbare Rolle.

Diese Diskussion wurde in der Bundesrepublik erst nach dem Irrflug eines Motorsegler-Piloten über der Frankfurter Innenstadt aufgegriffen, bei dem der hessische Innenminister sich an den Bundesverteidigungsminister gewendet hatte, der Kampfflugzeuge alarmieren ließ, obwohl die bereits eingesetzten Polizeihubschrauber die Situation unter Kontrolle hatten. Der Vorfall wurde damit erledigt, daß der offenbar geistig gestörte Pilot vom Tower des Frankfurter Flughafens zur Landung bewegt werden konnte.

\section{b) Neue Forderungen nach einem Einsatz der Streitkräfte im Inland}

Während die Bundesregierung den Einsatz der Bundeswehr im Inland auf die Abwehr eines terroristischen Angriffs mit einem Flugzeug beschränken wollte, griff die CDU/ CSU bei dieser ihr günstig erscheinenden Gelegenheit auf ihre alte Forderung zurück, die Bundeswehr im Inland »zur Aufrechterhaltung oder Wiederherstellung der öffentlichen Sicherheit und Ordnung « gegen terroristische Aktionen, sozusagen als Fortsetzung der Polizei mit militärischen Mitteln, vielfältig einsetzen zu können, etwa zum Schutz ziviler Objekte, aber auch zur Unterstützung der Polizei mit militärischen Mitteln, insbesondere zur allgemeinen Gefahrenabwehr bei irgendwelchen Angriffen aus der Luft oder von See.

Der unveränderte Art. 87a GG legt als Aufgabe der Streitkräfte ausdrücklich die Verteidigung fest und bestimmt darüber hinaus eindeutig, daß sie nur eingesetzt werden dürfen, wenn das Grundgesetz es ausdrücklich zuläßt. Damit sollte nicht nur die im Parlament und in der Öffentlichkeit verbreitete Sorge berücksichtigt werden, daß die Fähigkeiten der Bundeswehr zur Durchsetzung innenpolitischer Probleme mißbraucht werden könnten. Man war sich auch bewußt, daß die Bundeswehr eine Wehrpflichtarmee ist, deren militärischer Einsatz im Inland zu besonderen emotiona- 
len und politischen Konflikten führen muß. Es gehörte zum politischen Grundkonsens bei der sog. »Wiederaufrüstung «, daß diese Armee eben nur in völliger Übereinstimmung mit einer verfassungsändernden Mehrheit, also auf der Grundlage eines ausdrücklichen Verfassungsbeschlusses eingesetzt werden sollte.

\section{c) Deklaration als Amtshilfe durch Luftsicherheitsgesetz}

Ein solcher politischer Grundkonsens wurde bei der Beratung des hier angefochtenen Luftsicherheitsgesetzes nicht erzielt. Weil für keine der beiden Positionen eine verfassungsändernde Mehrheit zu erkennen war, setzte die Bundesregierung ihre Vorstellung streitig durch, indem sie den von ihr gewünschten Einsatz der Bundeswehr als Amtshilfe bei einer polizeilichen Aufgabe darstellte. Sie entwickelte das umfangreiche, mit dieser Verfassungsbeschwerde angefochtene Gesetz zur Neuregelung von Luftsicherheitsaufgaben und brachte es am 7. November 2003 über den Bundesrat in die Gesetzgebung ein. ${ }^{3}$

Der Bundespräsident hat das Gesetz trotz seiner verfassungsrechtlichen Bedenken gegen den Einsatz der Bundeswehr im Inland und gegen die mit dem Abschuß von Passagiermaschinen verbundene Tötung unschuldiger Menschen unterzeichnet und in seiner Presseerklärung Nr. 15/05 v. 12. Jan. 2005 eine verfassungsgerichtliche Prüfung angeregt. Das Gesetz ist am 15. Januar 2005 in Kraft getreten.

\section{Konkrete Gefahr für Leib und Leben der Passagiere im deutschen Luftraum}

Schon vor Inkrafttreten des neuen Gesetzes richteten Bund und Länder ein Alarmsystem ein, durch das für den Fall von Flügen, die als irregulär erscheinen - sog. »renegade flights « -, Alarmrotten strahlgetriebener Kampfflugzeuge alarmiert und an das Zivilflugzeug herangeführt werden. Die Zahl dieser als »alpha scramble« bezeichneten Fälle ist erstaunlich groß. Es handelt sich um mehrere Vorfälle pro Woche. Die Flugsicherung meldet vorsorglich jede Abweichung eines Flugzeugs von der angemel-

3 Der Bundesrat erklärte in seiner Sitzung v. 19. Dez. 2003, daß mit der umfangreichen Neuregelung der Luftsicherheitsaufgaben in Wirklichkeit keinerlei sicherheitlicher Gewinn verbunden sei. Sie führe vielmehr zu einer Verwirrung der Zuständigkeiten, sowie zu erheblichen Belastungen der mit der Luftsicherung befaßten Einrichtungen und Personen, ohne effizientere Verfahren einzuführen, vgl. dazu auch eingehend Meyer, ZRP 2004, S. 203 ff. Gegen die Regelung des Einsatzes der Bundeswehr wurden auch erhebliche verfassungsrechtliche Bedenken geltend gemacht, weil sie die verfassungsrechtlichen Vorgaben über die Amtshilfe sprenge (BRDrS. 827/1/03 ).

Die Bundesländer Bayern, Hessen, Sachsen und Thüringen stellten am 5. März 2004 einen weitgehenden verfassungsändernden Gesetzesantrag (BRDrS 181/04), der von der Bundestagsfraktion der CDU/CSU als Gesetzentwurf zur Änderung der Art. 35 und 87 a GG v. 9. März 2004 (BTDrS. 15/2649) übernommen, im Bundestag eingebracht und dort am 18. Juni 2004 von der Koalitionsmehrheit abgelehnt wurde (PIPr. 15/115).

Nachdem der Bundestag den Gesetzentwurf der Bundesregierung nach einer Anhörung am 26. Apr. 2004 im wesentlichen unverändert beschlossen hatte, wiederholte der Bundesrat in seiner Sitzung v. 9. Juli 2004 (BRDrS. 509/1/04 ) seine verfassungsrechtlichen und sachlichen Einwendungen. Nach erfolglosem Vermittlungsverfahren wies der Bundestag am 24. Sept. 2004 den Einspruch des Bundesrates vom selben Tag zurück. 
deten Flugroute oder den Abbruch des Funkkontakts einer Kontrollstelle, der sog. »NLFZ SiLuRa«, will heißen: »Nationales Lage- und Führungszentrum Sicherheit im Luftraum« in Kalkar. Dabei hat das Bundesministerium der Verteidigung auf Anfrage bestätigt, daß Abfangjäger schon vor Inkrafttreten des Gesetzes bis zu dreimal pro Monat an ein Zivilflugzeug herangeführt wurden, »um den Luftraum zu sichern« (vgl. Financial Times Deutschland v. 14. Jan. 2005). Das Jagdgeschwader $71 »$ Richthofen« hat von Jan. bis Nov. 2004 immerhin 250 Alarmfälle und 20 Starts angegeben (vgl. »Die Himmelspolizei«, Hamburger Abendblatt v. 25. 11. 2004).

Einzelne Vorgänge sind durch die Medien bekannt geworden, weil die Kampfflugzeuge der Bundeswehr von den überraschten Passagieren beobachtet und photographiert wurden - so bei einem LTU Charter Flug 139 der spanischen Fluglinie Futura am 19. Okt. 2004 nach Düsseldorf. Dabei handelte es sich möglicherweise um einen Fehler des Piloten, der die Maschine nicht rechtzeitig gemeldet haben soll (vgl. Bild, 20.10.2004, S. 6).

In einem anderen Fall wurde am 1. Juli 2004 die Entführung einer vom Täter nach München umgeleiteten Maschine ohne den Einsatz der bereits kreisenden bewaffneten Kampfflugzeuge auf andere Weise spektakulär beendet. Dort hat ein türkischer Passagier den mit einer Attrappe ausgerüsteten Entführer mit einem Fußtritt aus der geöffneten Tür des Flugzeugs auf das Rollfeld befördert. Der hatte sein Handy als Bombe ausgegeben (vgl. Süddeutsche Zeitung v. 1.7.2004, S. 36).

Der letzte bekannt gewordene Fall ereignete sich am 5. Jan. 2005 mit einer Boeing 737, FlugNr. 330X der dänischen Gesellschaft Maersk auf dem Flug von Lissabon nach Kopenhagen. Diese Maschine wurde von zwei Phantom-Jägern der Bundeswehr abgefangen und längere Zeit begleitet, weil der Funkkontakt abgerissen war. Bei den Militärpiloten soll höchste Unruhe geherrscht haben, weil sie zu den Piloten des Zivilflugzeugs weder Funk- noch - wegen der Lichtverhältnisse - Blickkontakt herstellen konnten (vgl. Bild v. 18.1.2005). Erst später stellte sich heraus, daß die Unterbrechung des Funkkontakts über 45 Minuten technische Gründe hatte. Die Zivilpiloten hatten sich vollkommen einwandfrei verhalten. Bei Abbruch des Funkkontaktes müssen sie nach den internationalen Vorschriften entsprechend der zuvor erteilten Freigabe zum Zielflughafen weiterfliegen.

Wenn auch die mit der Verfassungsbeschwerde angefochtenen Bestimmungen bis jetzt noch nicht zum Abschuß eines Passagierflugzeugs über der Bundesrepublik geführt haben, so folgt daraus nicht, daß das nur eine entfernte, eigentlich nicht ernst zu nehmende Möglichkeit sei. Bei einem Flug über der Bundesrepublik besteht nicht nur bei einer Flugzeugentführung durch Terroristen, einen »normalen « Straftäter oder einen Irren, sondern schon bei einem Pilotenirrtum oder einem Instrumentenfehler die konkrete Gefahr für Leib und Leben eines jeden Passagiers, Objekt eines Angriffes der Bundeswehr zu werden. Es soll dann auf die Nervenstärke, die Entschlußkraft und die praktische Vernunft des jeweiligen Verteidigungsministers der Bundesrepublik - oder seines Vertreters im Amt - und natürlich auf die ihm vorgetragenen Informationen ankommen, ob er die Maschine abschießen läßt oder nicht. 


\section{Verunsicherung von Bundeswehr-Piloten}

Die dargestellte gesetzliche Regelung und ihre bisherige Handhabung im Luft-raum der Bundesrepublik haben auch unter den Piloten der Bundeswehr zur Beunruhigung geführt. Das ergibt sich nicht nur aus der Befürchtung, eines Tages den Befehl zu erhalten, ein vollbesetztes entführtes Linienflugzeug abschießen zu sollen. Auch für die Piloten der Bundeswehr stellen sich existentielle Fragen angesichts der ethisch-moralischen Zumutung, aber auch angesichts der politischen Diskussion über die vom Bundestag beschlossene gesetzliche Regelung und wegen der drastischen technischen Mängel der bisherigen Ausrüstung der Kampfflugzeuge für die zunächst geforderte Warnung des zivilen Flugzeugs, das notfalls abgeschossen werden soll. Es ist streitig geblieben, ob ein solcher Befehl verfassungskonform und rechtmäßig wäre und welche Rechtsfolgen sich für den Kampfpiloten ergeben, der auf Befehl unschuldige Menschen tötet oder die Ausführung eines solchen Befehls verweigert (vgl. dazu »Jet News«, Organ des Verbandes der Besatzungen strahlgetriebener Kampfflugzeuge der Deutschen Bundeswehr e.V., Nr. 2/2004, S. 13 ff.). ${ }^{4}$

Die Piloten der Zivilflugzeuge in der Vereinigung Cockpit haben sich mit Schreiben v. 7. Dez. 2004 an den Bundespräsidenten mit der dringenden Bitte gewendet, dieses Gesetz nicht auszufertigen. Auch der Verband der Allgemeinen Luftfahrt e.V. AOPA (Aircraft Owners and Pilots Association) hat eindringlich vor den Folgen des als verfassungswidrig betrachteten Gesetzes gewarnt (vgl. AOPA-letters 6/03, S. 8).

\section{Menschenleben im Rechtsstaat: weder Abwägung noch Opferung}

Bei dem Abschuß eines Flugzeugs sind die mit ihm entführten Insassen nicht nur bedauerliche »Kollateralschäden«, die man eigentlich nicht gemeint hat. Sie sind vielmehr unmittelbares Objekt eines staatlichen Angriffs auf ihr Leben, wenn der Minister meint, das sei im Ergebnis für alle besser so. Sie werden geopfert. Das gilt unabhängig davon, ob der Verteidigungsminister seine Entscheidung auf einer gesicherten Grundlage trifft, ob er sich dabei etwa auf die Erklärung eines Entführers stützt, die ihn zu der Annahme führt, daß das Flugzeug »gegen das Leben von Menschen« eingesetzt werden soll und daß die an Bord befindlichen Passagiere keine Möglichkeit mehr haben, sich selbst zu befreien. Welche Werte er gegeneinander abzuwägen hat, läßt das Gesetz offen.

Ein Rechtsstaat kann seine Aufgabe, das Leben seiner Bürger zu schützen, nicht dadurch erfüllen, daß er die Opfer einer Straftat vorsätzlich tötet. Niemand kann sagen, wieviele Menschen möglicherweise durch die Angreifer tatsächlich ihr Leben verlieren würden und sie von Rechts wegen numerisch abwägen gegen das Leben der bei einem Abschuß mit Sicherheit getöteten Passagiere, der Besatzung der Maschine und

4 Dabei betonen z. B. die Piloten des Jagdgeschwaders 71, daß sie auf Befehl auch 200 bis 300 Passagiere abschießen würden. Man müsse professionell darauf vertrauen, daß das System funktioniere. »Das System hat Anspruch auf Gehorsam. «Dabei meinen sie, daß sie »wohl straffrei« ausgehen, offen seien aber zivilrechtliche Ansprüche und ob ihre Familien darunter leiden könnten? (vgl. Hamburger Abendblatt a.a.O.). 
der an dem gesamten Vorgang völlig unbeteiligten Menschen, die möglicherweise am Boden von dem abgeschossenen Flugzeug getroffen werden. Das Leben ist keine Sache und kein Vermögenswert. Niemand weiß, wann und wie es endet. Sein Wert hängt nicht von seiner vermuteten Dauer ab. Die Verfassung erlaubt dem Staat nicht, gefährdetes Leben gering zu schätzen. Man verliert nicht das Recht auf Leben, weil der Verteidigungsminister oder sein Vertreter im Amt meint, es würde nicht mehr lange dauern und es sei für alle besser so.

Wir sind möglicherweise bedroht, sei es von Terroristen, von »normalen« Verbrechern oder von Geisteskranken. Aber wir befinden uns nicht im Krieg.

\section{Zur Begründetheit der Verfassungsbeschwerde}

\section{Verletzung der Grundrechte auf Menschenwürde und Leben in ihrem Wesensge- halt}

Die Beschwerdeführer werden durch die angefochtenen Bestimmungen des LuftSiG in ihren Grundrechten auf Menschenwürde und Leben gem. Art. 1 Abs. 1, Art. 2 Abs. 2 GG verletzt. Diese Grundrechte werden entgegen Art. 19 Abs. 2 GG in ihrem Wesensgehalt angetastet.

a) Herabwürdigung des Menschen zum bloßen Objekt staatlichen Handelns

Die Beschwerdeführer werden durch das angefochtene Gesetz zum bloßen Objekt staatlichen Handelns gemacht. Der Wert und die Erhaltung ihres Lebens wird durch die $\S \S 13,14$ und 15 LuftSiG in das Ermessen des Verteidigungsministers unter mengenmäßigen Gesichtspunkten und nach der ihnen »den Umständen nach « vermutlich verbliebenen Lebenserwartung gestellt. Die Beschwerdeführer sollen im Ernstfall geopfert und vorsätzlich getötet werden, wenn der Verteidigungsminister auf der Grundlage der ihm zu diesem Zeitpunkt vorliegenden Informationen annimmt, daß ihr Leben nur noch kurze Zeit dauern wird und daher im Vergleich zu den sonst drohenden Verlusten keinen Wert mehr habe oder jedenfalls nur noch minderwertig sei.

Da der Verteidigungsminister im Zeitpunkt der Entscheidung weder die Menschen kennt, die er retten möchte, noch die anderen, die er dafür opfern müßte, kann er nur eine mengenmäßige oder materielle Abwägung treffen, welche Maßnahme wohl das kleinere Übel sei. Auch die eingesetzten Soldaten können keine wie immer denkbare Abwägung treffen und sollen das auch gar nicht. Sie bekommen den Befehl, die Opfer zu töten, und es wird von ihnen erwartet, daß sie das dann ohne Zögern tun.

\section{b) Kaschierung der Abwägung Leben gegen Leben}

Die von dem Verteidigungsminister zu treffende Entscheidung wird im Gesetz dadurch verdeckt, daß der Wortlaut des § 14 LuftSiG den Eindruck erweckt, es gehe um die Abwägung des Lebens von Menschen gegen den Abschuß eines »Luftfahrzeugs«, also um die Zerstörung einer Maschine. Selbst der Abschuß des Flugzeugs wird beschönigend als »unmittelbare Einwirkung mit Waffengewalt« umschrieben. Im Gesetzeswortlaut wird nicht einmal erwähnt, ob sich in dem Luftfahrzeug Menschen befin- 
den oder nicht. Sie kommen nur noch in der Begründung als ebenfalls gefährdete Personen vor. Der Gesetzgeber hat sie bereits abgeschrieben. Der Bundesinnenminister hat in seiner Pressekonferenz v. 12. Jan. 2005 ausdrücklich erklärt, es gehe gar nicht um die Abwägung »Leben gegen Leben«, weil die Passagiere ja eigentlich schon so gut wie tot seien. Auch die Menschen, die durch den Abschuß »des Luftfahrzeugs« über der dicht besiedelten Bundesrepublik am Boden in den Tod gerissen werden können, weil die Absturzstelle nicht berechenbar ist, kommen weder im Gesetz, noch in seiner Begründung vor. Es versucht, die häßliche Wahrheit zu kaschieren, daß eine unbestimmte Vielzahl von Menschen mit dem vorsätzlichen Abschuß des Flugzeugs von der Bundeswehr getötet werden soll, wenn der Verteidigungsminister »den Umständen nach « annimmt, daß die Entführer des Flugzeugs sonst das Leben anderer Menschen gefährden, von denen er hofft, sie auf diese Weise retten zu können. Das Gesetz begnügt sich damit, eine Entscheidung nur über die Frage zu fordern, ob das Luftfahrzeug »den Umständen nach « gegen das Leben von Menschen eingesetzt werden soll und ob das auf andere Weise als durch »unmittelbare Waffengewalt« abgewehrt werden kann. Da wird ein Menschenopfer für einen Zweck gebracht, von dem der Verteidigungsminister oder sein Vertreter im Amt »den Umständen nach annimmt «, daß er wichtiger ist als die zu tötenden Menschen. Dabei wird in Kauf genommen, daß der Verteidigungsminister in den wenigen Minuten, die ihm im Ernstfall für seine Entscheidung zur Verfügung stehen würden, weder die Richtigkeit der ihm dargestellten Sachlage, noch eine eigene sachlich begründete Abwägung treffen kann. Er muß ebenso, wie es die Piloten des Jagdgeschwaders 71 für sich gesagt haben, darauf vertrauen, »daß das Gesamtkonzept funktioniert«. Wer eigentlich »das System « ist, das die Entscheidung über Leben und Tod in Wirklichkeit trifft, bleibt anonym. In der Begründung der $\S \S 13,14$ LuftSiG kommt das alles nicht vor. Der Vorgang wird wie die Fortsetzung einer polizeilichen Maßnahme durch das Militär und den in Friedenszeiten als »Inhaber der Befehlsgewalt« zuständigen Verteidigungsminister abgehandelt. Das ist absurd.

Diese Kaschierung des wirklichen Regelungsinhalts ist mit dem Wunsch, die Handlungsfreiheit des Staates zu erhalten, nicht zu begründen. Die Verfasser des Gesetzestextes hatten nicht den politischen und rechtlichen Mut, ausdrücklich zu sagen, daß der Verteidigungsminister berechtigt werden soll, mit seiner Entscheidung die Tötung von Menschen gegen die vermutete Gefährdung anderer Menschen abzuwägen. Aber in der Sache soll das so sein. Das bestätigt die Mißachtung der Opfer, auf der das Gesetz beruht. Sie werden ohne jede eigene Schuld in den Tod geschickt, weil sie sich unglücklicher Weise mit - in der Vorstellung des Verteidigungsministers - zeitlich nur noch begrenzter Lebensdauer in einem als Gefahr eingestuften Luftfahrzeug oder am Boden an der falschen Stelle befinden.

Wenn der Gesetzgeber nicht einmal den Mut zu einer klaren Aussage hat, dann fragt sich auch, wie denn eigentlich die Soldaten der Bundeswehr, mögen es selbst Piloten oder mag es Bedienungspersonal von Flugabwehrgeschützen oder von Boden-LuftRaketen sein, den Mut haben sollen, eine Passagiermaschine mit einer unbestimmten Anzahl von Männern, Frauen und Kindern an Bord über dem dichtbesiedelten Gebiet der Bundesrepublik abzuschießen. Dieses verfassungswidrige Gesetz gibt ihnen weder 
einen moralischen, noch einen rechtlichen Schutz bei der von ihnen selbst zu treffenden Entscheidung, ob sie einen auf dieser Grundlage erteilten Befehl befolgen wollen.

c) Keine Legitimation durch Vorentscheidungen des Bundesverfassungsgerichts

Das angerufene Gericht hat in seiner - nur sehr knapp begründeten - Schleyer-Entscheidung vom 16. 10. 1977 (NJW 1977, 2255) eindringlich darauf hingewiesen, daß der Staat nach Art. 2 Abs. 2 S. 1 GG in Verbindung Art. 1 Abs. 1 S. 2 GG verpflichtet ist, $\mathrm{j}$ e $\mathrm{d}$ e $\mathrm{s}$ menschliche Leben zu schützen und daß das menschliche Leben einen Höchstwert darstellt, dessen Erhaltung eine besonders ernst zu nehmende Verpflichtung ist. Die Verfassung begründe diese Schutzpflicht nicht nur gegenüber dem Leben des Einzelnen, sondern auch gegenüber der Gesamtheit seiner Bürger. Der Staat müsse gerade bei terroristischen Angriffen eine weite Handlungsfreiheit bei seiner Entscheidung haben, wie das Leben zu schützen sei. Man könne also aus der Verfassung nicht generell eine bestimmte Verhaltensweise herleiten, die der Staat bei seiner Entscheidung wählen müsse. Mit keinem Wort deutet diese Entscheidung aber an, daß der Staat seine Verpflichtung, das Leben eines jeden Einzelnen zu schützen, dadurch erfüllen dürfe, daß er eine Minderheit der von ihm zu schützenden Bürger vorsätzlich tötet.

Bei der Entscheidung des Gerichts ging es darum, ob es eine rechtliche Verpflichtung des Staates gebe, zur möglichen Rettung des Lebens des Hanns Martin Schleyer den Entführern nachzugeben, die von ihnen genannten Strafgefangenen freizulassen und dabei im Interesse der Rettung Schleyers in Kauf zu nehmen, daß die freigepreßten Terroristen alsbald weitere Straftaten begehen können. In diesem Zusammenhang hat das Gericht dem Staat die notwendige Entscheidungs- und Handlungsfreiheit zur Rettung menschlichen Lebens eingeräumt. Es sollte dem Staat überlassen bleiben, auf welchem Wege er versuchen will, seine verfassungsrechtliche Pflicht zur Rettung des Verbrechensopfers Schleyer zu erfüllen. Es ging also gerade nicht etwa darum, ob es dem Staat erlaubt sei, den Hanns Martin Schleyer zu töten, um andere Leben zu retten.

So liegt es aber im vorliegenden Fall. Dem angefochtenen Gesetz geht es nicht darum, welche Mittel der Staat anwenden darf oder muß, um das Leben der entführten Passagiere zu retten. Vielmehr will der Staat sich mit dem angefochtenen Gesetz das Recht nehmen, die entführten Passagiere mitsamt den Tätern zu töten, um andere möglicherweise gefährdete Personen zu schützen.

Selbst wenn für die Bekämpfung von Terroristen abweichende Maßstäbe zulässig wären, würde das an der Verfassungswidrigkeit der $\S \S 13,14,15$ LuftSiG nichts ändern, weil sich das angefochtene Gesetz nicht nur auf die im Schleyer-Urteil gewürdigten besonderen Umstände eines terroristischen Angriffs bezieht. Es soll auch Flugzeugentführungen oder Sabotageakte erfassen, die »von Kriminellen ohne politische Absichten oder geistig verwirrte Einzeltäter verursacht werden«, wie sich aus $§ 1$ LuftSiG und der Allgemeinen Begründung des Gesetzentwurfes ausdrücklich ergibt.

d) Unvereinbarkeit des „finalen Rettungstotschlags“ mit der Rechtsordnung des Grundgesetzes

Unsere Rechtsordnung, wie sie sich übereinstimmend aus den Polizeigesetzen des Bundes und der Länder ergibt, erlaubt es zwar, im äußersten Notfall einen Täter zu tö- 
ten, wenn dadurch ein unmittelbar von ihm bedrohter Mensch gerettet werden kann. Es ist auch erlaubt, im äußersten Notfall auf einen Menschen in einer Menschenmenge zu schießen und dabei Unbeteiligte zu gefährden. Es ist aber in keinem Fall zulässig, auf eine Menschenmenge zu schießen oder Unbeteiligte vorsätzlich zu töten oder, wie hier beabsichtigt, die gesamte Menschenmenge einschließlich der Täter zu töten. ${ }^{5}$

Eine Abwägung »Leben gegen Leben« nach dem Maßstab, wieviele Menschen es möglicherweise auf der einen und wieviele es auf der anderen Seite sind, ist unzulässig. Es entspricht der ständigen Rechtsprechung des BVerfG, daß der Schutz des einzelnen Lebens nicht wegen des Ziels aufgegeben werden darf, andere Leben zu retten: »Jedes menschliche Leben ist als solches gleich wertvoll und kann deshalb keiner irgendwie gearteten unterschiedlichen Bewertung oder gar zahlenmäßigen Abwägung unterworfen werden $\ll{ }^{6}$

Die Menschenwürde und das menschliche Leben sind die höchsten Werte, die unserer Rechtsordnung zugrunde liegen und zu deren Schutz der Staat berufen ist. ${ }^{7}$

Er hat eine Ermessensfreiheit und Handlungsfreiheit in der Frage, wie er das Leben eines jeden Einzelnen schützt und zu schützen vermag. Er darf Menschen gefährden, um einen anderen Menschen zu retten. Aber er darf Menschen nicht deswegen töten, weil es weniger sind, als er durch ihren Totschlag zu retten hofft.

Auch der Gesetzesvorbehalt in Art. 2 Abs. 2 S. 3 GG führt zu keinem anderen Ergebnis. Denn er steht unter dem Vorbehalt des Art. 19 Abs. 2 GG. Die Garantie des Wesensgehaltes läßt eine Sonderregelung durch den einfachen Gesetzgeber nur begrenzt zu. Die Garantie ist selbst Teil der Verfassung, sodaß die Grundrechte eben nicht unter Berufung auf Sonderlagen oder Interessenabwägungen in das Ermessen des Gesetzgebers gestellt sind. Das gilt in besonderer Weise für das Recht auf Leben, weil der Schutz vor physischer Vernichtung grundsätzlich keine geringere Bedeutung hat als die Achtung der Menschenwürde. Die Begrenzung des Lebensrechts bedeutet eben immer den Tod. Der Wesensgehalt eines Grundrechts wird jedenfalls dann berührt, wenn der Mensch zum bloßen Objekt staatlichen Handelns gemacht, also wenn, wie hier, das Grundrecht auf Leben durch Voraussetzungen verwehrt wird, auf deren Erfüllung der Einzelne bei allen Mühen keinen Einfluß hat. Der Staat darf zwar die Gefährdung menschlichen Lebens zur Verteidigung der Rechtsordnung in Kauf nehmen. Er darf unter Umständen auch fordern, das Leben zu wagen. Aber der Staat des Grundgesetzes darf nicht von Rechts wegen ein vorsätzliches Menschenopfer fordern. Die Tötung eines Opfers, die »Finalität im volitiven Sinn « ist ihm verwehrt. ${ }^{8}$

Der finale Rettungstotschlag ist mit unserer Verfassung, mit unserem Rechtsdenken, mit unseren Traditionen unvereinbar. Das Leben von Menschen kann nicht gegenseitig wie ein materieller Wert nach Zahl und Dauer gegeneinander aufgerechnet und abgewogen werden.

5 Vgl. dazu im einzelnen Rachor in Lisken-Denninger, Handbuch d. Polizeirechts, S. 554 ff. und die dortigen umfangreichen Zitate.

6 Vgl. dazu auch besonders ausführlich und eindringlich Baumann, »Das Grundrecht auf Leben unter Qualifizierungsvorbehalt?« DÖV 2004, 853 ff. (861).

7 Vgl. BVerfGE 49, 24 (53); 46, 160 (164); auch Maunz-Dürig, Rdnr. 22 zu Art. 1 I GG.

8 Vgl. schon Dürig in AöR 56, 117 ff.; Maunz-Dürig, Rdnr. 6, 18 zu Art. 19 Abs. 2 GG, Rdnr. 34 zu Art. 2 Abs. 2 GG und die dort zitierte a.o. umfangreiche Literatur. 
Auch der Hinweis auf den in $\S 34$ StGB enthaltenen Rechtsgedanken des rechtfertigenden Notstands führt zu keinem anderen Ergebnis. Dabei mag hier dahingestellt bleiben, daß eine ausdrückliche Berufung auf $\S 34$ StGB da ausscheidet, wo eine besondere gesetzliche Regelung getroffen worden ist. Der $\S 34$ StGB ist kein Auffangtatbestand für den Fall der Verfassungswidrigkeit der hier angefochtenen gesetzlichen Regelung der $\S \S 13$ ff. LuftSiG. Aber unabhängig davon würde auch der Rechtsgedanke des § 34 StGB eine Tötungshandlung grundsätzlich nicht rechtfertigen, und zwar auch dann nicht, wenn durch die Tötung eines Menschen eine größere Zahl anderer Menschen gerettet werden würde. Das gilt auch dann, wenn die getöteten Menschen todgeweiht waren. Der Gesetzgeber kann das Verbot nicht aufheben, die Hand an das Leben unschuldiger Menschen zu legen. ${ }^{9}$

Ein Rechtssatz, daß ein Leben nur noch einen geringen oder keinen Wert mehr hat, wenn es nach menschlichem Ermessen bald endet, hätte auf vielen Rechtsgebieten eine verheerende Wirkung. Die Auffassung, die der Bundesinnenminister in seiner Pressekonferenz vom 12. Januar 2005 vorgetragen hat, es gehe nicht um die Abwägung Leben gegen Leben, weil die Passagiere des Flugzeugs eigentlich schon tot seien, ist ein unerträglicher Ausdruck der Mißachtung, der Geringschätzung, der Abwertung dieser Menschen, als ob man sich erstaunt fragen müßte, was die denn eigentlich noch wollen. Mit derselben Betrachtungsweise könnte man einen moribunden Patienten zu Tode bringen, um ein möglichst frisches Organ bei einer Transplantation einsetzen zu können. Unter der Herrschaft des Grundgesetzes kann es nicht Bestandteil unserer Rechtsordnung werden, daß irgend jemand, mag er der Bundesregierung angehören oder nicht, aus Gründen der Opportunität über das Leben anderer Menschen verfügt, mag es wohlmeinend sein oder nicht.

Die Bundesregierung hat, wie alle anderen Menschen auch, nicht die Fähigkeit zu wissen, wie lange das Leben der Passagiere eines Luftfahrzeugs dauert. Und sie hat nicht das Recht, das Leben dieser Passagiere als minderwertig im Vergleich zum Leben anderer Menschen zu behandeln und sie deswegen zu töten.

Auf dieser Vorstellung beruht die hier angefochtene gesetzliche Regelung. Sie verstößt in krasser Weise gegen die Menschenwürde und das Recht auf Leben. Die nach Art. 2 Abs. 2 GG mögliche Beschränkung des Rechts auf Leben ist daher hier auch ein Verstoß gegen Art. 1 und 19 Abs. 2 GG und mit dem Grundgesetz nicht vereinbar.

\section{Verletzung des Bestimmtheitsgebotes}

Die gesetzliche Regelung entbehrt auch der für einen so tiefen Eingriff in verfassungsmäßige Rechte notwendigen Bestimmtheit.

a) Wesentlichkeitsgrundsatz als Inpflichtnahme des Gesetzgebers

Die Anforderungen an die von der Verfassung geforderte Normenklarheit sind um so strenger, je höher das Rechtsgut ist, in das eingegriffen werden soll, und um so wesentlicher die Folgen administrativen Handelns für den Betroffenen sind.

9 Vgl. Schönke/Schröder, Rdnr. 34, 23, 24 zu § 34 StGB mit zahlr. Zitaten und BGHSt 35, 350. 
Dabei ist zwar nicht zu beanstanden, wenn sich der Gesetzgeber unbestimmter Rechtsbegriffe bedient, die der juristischen Auslegung zugänglich sind. Der Gesetzgeber darf aber nicht durch formelhafte Wendungen eine Entscheidung, die er nach dem Wesentlichkeitsgrundsatz selbst zu treffen hat, in Wirklichkeit dem Ermessen der ausführenden Verwaltung überlassen. Das hat das BVerfG bereits in dem sog. ElfesUrteil v. 16. 1. 1957 (BVerfGE 6, 32 ff., NJW 1957, 297 ff.) ausgeführt und zuletzt durch die Entscheidung v. 3. 3. 2004 zur Überwachung des Briefverkehrs und der Telekommunikation im Rahmen des Außenwirtschaftsrechts (BVerfG NJW 2004, 2213 ff.) bekräftigt.

b) Unbestimmtheit von Normen im Luftsicherheitsgesetz

Es mag dahingestellt bleiben, daß das Gesetz nicht präzisiert, wann ein »erheblicher Luftzwischenfall« vorliegt, der zu einem besonders schweren Unglücksfall i. S. des Art. 35 Abs. 2 bzw. 3 GG führen kann.

Es soll auch dahingestellt bleiben, ob die »unmittelbare Einwirkung von Waffengewalt« tatsächlich nur gem. § 14 Abs. 4 LuftSiG vom Bundesminister der Verteidigung angeordnet werden kann oder auch von dem vom Minister nach § 15 Abs. 3 LuftSiG ermächtigten Inspekteur, der seinerseits alle Maßnahmen nach $§ 15$ Abs. 1, 14 Abs. 1 und 4 LuftSiG anordnen kann, zu denen auch die unmittelbare Einwirkung von Waffengewalt gehört. Dabei geht es nicht nur um die politische Verantwortung, sondern gegebenenfalls auch um die Rechtmäßigkeit eines vom Inspekteur an die eingesetzten Piloten oder an sonstige Luftabwehrkräfte erteilten Befehls.

Nicht akzeptabel ist aber die Unbestimmtheit der Regelung des $§ 14$ Abs. 3 LuftSiG. Nach ihrem Wortlaut hat der Verteidigungsminister als ultima ratio nur die Fortexistenz einer gefährlichen Maschine gegen das Leben von ihr voraussichtlich gefährdeter Menschen abzuwägen. Der Gesetzgeber unterläßt es, auch nur anzudeuten, nach welchem Maßstab sich der Verteidigungsminister bei seiner Entscheidung über Leben und Tod richten soll. Genügt die Gefährdung des Lebens eines einzelnen Menschen oder müssen es mehrere sein? Kommt es auf die Zahl der Insassen des Flugzeugs im Verhältnis zur Zahl der Personen an, gegen deren Leben das Flugzeug voraussichtlich eingesetzt werden soll oder hat der Verteidigungsminister vielleicht sogar einen anderen Maßstab dafür, wie bedeutungsvoll das Leben der einen oder der anderen Seite einzuschätzen ist? Wird das Luftfahrzeug gegen das Leben von Menschen eingesetzt, wenn sie nicht unmittelbar durch den Absturz der Maschine getötet werden, sondern erst mittelbar, indem sie etwa erst durch die Auswirkungen eines Absturzes auf eine Chemische Anlage oder auf ein Kernkraftwerk in Lebensgefahr geraten? Muß es sich also um eine gegenwärtige und unmittelbare Gefahr für die dabei zu Tode kommenden Menschen handeln oder können auch die möglichen Spätfolgen berücksichtigt werden, die durch den Absturz eines Flugzeugs in Zukunft entstehen können? Müssen die »Umstände «, nach denen »davon auszugehen ist «, daß das Luftfahrzeug gegen das Leben von Menschen eingesetzt werden soll, auf feststehenden Tatsachen beruhen oder genügen tatsächliche Annahmen oder begründete Vermutungen? Ist der Abschuß einer Maschine und die Tötung ihrer Insassen »das einzige Mittel zur Abwehr dieser gegenwärtigen Gefahr«, wenn die Entführer eine finanzielle Forderung stellen oder die Ab- 
gabe einer Erklärung über das Fernsehen verlangen? Wie darf sich der Verteidigungsminister entscheiden, wenn die Entführer einer mit einer Vielzahl von Personen besetzten Maschine erklären, sie wollten sie zwar auf das Reichstagsgebäude stürzen, aber dabei so viel Zeit einräumen, daß er vollkommen evakuiert werden könnte? Sicher scheint nur zu sein, daß das Flugzeug nicht abgeschossen werden muß, sondern daß der Verteidigungsminister sich durchaus entscheiden kann, die Folgen eines Absturzes hinzunehmen. Er wird im Wortsinn zum Herrn über Leben und Tod und darf von Rechts wegen nach Opportunität entscheiden, ob die einen oder die anderen sterben sollen. Wer meint, das gesetzlich regeln zu können, sollte etwas präziser werden müssen. Was heißt: »wenn nach den Umständen davon auszugehen ist «, daß das Luftfahrzeug gegen das Leben von Menschen eingesetzt werden soll? Müßte eine solche Annahme nicht wenigstens auf konkreten Tatsachen beruhen und die unmittelbare Gefahr eines Ereignisses vom Umfang einer Naturkatastrophe drohen, wenn man dem Gedanken überhaupt folgen wollte? Eine Entscheidung über eines der höchsten Rechtsgüter, das Leben, kann nicht in das Ermessen eines einzelnen Mitglieds der Bundesregierung gestellt werden, das dem Parlament gegenüber nicht einmal unmittelbar verantwortlich ist.

\section{Verfassungswidriger Einsatz der Bundeswehr im Inland durch Luftsicherheitsge- setz}

Die Beschwerdeführer sind in ihrem Grundrecht auf Leben auch dadurch beeinträchtigt, daß das zu seiner Einschränkung beschlossene LuftSiG wegen Verstoßes gegen Art. 87a GG verfassungswidrig ist. Wenn auch Art. 87a GG kein mit der Verfassungsbeschwerde angreifbares Grundrecht der Beschwerdeführer gem. § 90 BVerfGG ist, so sind sie doch in ihren Grundrechten auf Leben und Menschenwürde auch dadurch verletzt, daß der Einsatz der Bundeswehr im Inland auf der Grundlage der $\S \S 13$ ff. des angefochtenen Gesetzes verfassungswidrig wäre.

\section{a) „Verteidigung“ als Aufgabe der Streitkräfte gemäß Art. 87a GG}

Art. 87 a GG bestimmt als Aufgabe der Streitkräfte die Verteidigung. Außer zur Verteidigung ist der Einsatz der Streitkräfte gem. Art. 87a Abs. 2 GG nur dann zulässig, wenn das Grundgesetz es ausdrücklich zuläßt. Die Verfassung verlangt also die Zulassung mit klaren, eindeutigen Worten. Eine mehr oder weniger teleologische Auslegung oder die zielbewußte interpretatorische Ausfüllung einer angeblichen Lücke wird dem ausdrücklichen Regelungsinhalt des Art. 87a Abs. 2 GG nicht gerecht. Es trifft zwar zu, daß die Wehrverfassung der Bundesrepublik infolge ihrer historischen Entwicklung lückenhaft ist. Das ändert aber nichts daran, daß Art. 87a Abs. 2 GG verlangt, den Inhalt der Verfassung nicht durch neue Interpretationen, sondern durch neue Formulierungen zu ändern, wenn man der Bundeswehr neue Aufgaben übertragen will. Der Gesetzgeber unterliegt hier dem Gebot strikter Texttreue. Die möglichen Verwendungszwecke der Bundeswehr im Innern sollten im Grundgesetz abschließend aufgezählt sein. Der Verfassungsgesetzgeber wollte bewußt dem einfachen Gesetzgeber die Möglichkeit nehmen, sich auf neue Notlagen ohne Verfassungsänderung zu berufen. ${ }^{10}$ 
aa) Die Regelung der $\S \S 13,14$ und 15 LuftSiG betreffen keinen Fall einer Verteidigung i. S. des Art. 87a GG. Unter Verteidigung versteht man die Abwehr eines Angriffs von außen durch einen anderen Staat mit militärischen Mitteln. Diese Voraussetzungen liegen offenkundig nicht vor. Die Regelungen beziehen sich - wie oben ausgeführt - nicht nur auf Terroristen, sondern auch auf Flugzeugentführungen und Sabotageakte aus »normalen« kriminellen Motiven oder als Folge einer geistigen Störung.

Auch Terroristen sind Kriminelle. Sie unterliegen dem Strafrecht und nicht der Genfer Konvention. Terroristische Straftaten sind für das innerstaatliche Recht Straftaten und keine militärischen oder kriegerischen Handlungen. Das gilt auch für Flugzeugentführungen. Sie sind auf Grund des Tokioter Abkommens v. 14. Sept. 1963 (BGB1. 1969 II, S. 121) durch § 316c StGB ausdrücklich zu einem Straftatbestand gemacht worden, um ihnen als Verbrechen entsprechend begegnen zu können.

Daran ändert auch nichts, daß es eine breite völkerrechtliche Diskussion darüber gibt, ob ein Staat nach Art. 51 der Charta der Vereinten Nationen oder nach völkerrechtlichem Gewohnheitsrecht ein Selbstverteidigungsrecht gegen eine terroristische Organisation oder gegen einen Staat hat, der entweder Terroristen auf seinem Gebiet zu Aktionen gegen den anderen Staat ermutigt oder nichts dagegen unternimmt, daß Terroristen auf seinem Gebiet Angriffshandlungen gegen den anderen Staat planen und vorbereiten (vgl. dazu auch die vorsichtigen Formulierungen der Erklärung des Bündnisfalles der NATO v. 2. Okt. 2001, Blätter f. deutsche u. internationale Politik 01, S. 1403). Nach noch herrschender Meinung hat sich trotz einer Reihe militärischer Vorgänge ein solches völkerrechtliches Gewohnheitsrecht noch nicht gebildet. ${ }^{11}$

Hier kommt es jedenfalls nicht darauf an, ob die Bundesrepublik völkerrechtlich berechtigt ist, unter bestimmten Umständen das Eindringen eines fremden Flugzeugs in den Luftraum der Bundesrepublik mit militärischen Mitteln zu verhindern, so wie etwa die damalige Sowjetunion am 1. Sept. 1983 die koreanische Boeing 747 - Flug KAL 007 - wegen des unbefugten Eindringens in den nationalen Luftraum und des Überfliegens eines militärischen Sperrgebietes abgeschossen hat und dabei die 269 Passagiere und Besatzungsmitglieder tötete. ${ }^{12}$

10 Vgl. BTDrS. V/2872, S. 2; BVerfGE 90, 286 (357); besonders eindringlich Linke, DÖV 2003, 890 ff. (893); Tettinger, Anhörungsgutachten, ADrs.15 (4)102 C, S. 5; Stern, Staatsrecht, Bd, 1, 1984, S. 160 ff.; Baldus, NVerwZ 2004, 1282.

Vgl. auch Kutscha, »Verteidigung - Vom Wandel eines Verfassungsbegriffs«, KJ 2004, 228 ff., der nach detaillierter Darstellung der Entwicklung der Wehrverfassung das hier angefochtene Gesetz zutreffend als »Musterbeispiel legislativer Irreführung « bezeichnet.

11 Es kann darum hier auch dahingestellt bleiben, daß selbst für den Kriegsfall Art. 51 Abs. 4 des Zusatzprotokolls v. 12. Dez. 1977 zum Genfer Abkommen (BGBl. 1990 II, 1551 ff.) einen militärischen Angriff verbietet, der unterschiedslos Kombattanten und Zivilbevölkerung trifft.

12 Der Vorgang führte zu einer Sondersitzung des UN-Sicherheitsrates und dem ICAO Beschluß eines Art. 3 bis des Chicagoer Abkommens, in dem sich die Vertragsstaaten verpflichteten, unbeschadet ihrer Rechte aus der UN Charta keinerlei Waffengewalt gegen ein sich in der Luft befindliches Zivilflugzeug anzuwenden und die Sicherheit der Maschine und der Passagiere nicht zu gefährden, vgl. Ipsen, Völkerrecht, 1999, S. 804. 
Der Regelungsinhalt der mit der Verfassungsbeschwerde angegriffenen $\S \S 13,14$ und 15 LuftSiG bezieht sich auf jedes Luftfahrzeug, das sich im Luftraum der Bundesrepublik unabhängig davon befindet, ob es aus dem Ausland eingeflogen oder im Inland gestartet ist, und unabhängig davon, ob es als Werkzeug eines terroristischen Angriffs eingesetzt werden soll, ob es in einer anderen kriminellen Motivation entführt wurde, oder ob es sich um einen geisteskranken Täter handelt. Dementsprechend bezieht sich die amtliche Begründung des Gesetzentwurfes auch nicht auf eine nach Art. 87a GG zugelassene Verteidigung durch die Streitkräfte, sondern bemüht sich darum, den Einsatz als eine polizeiliche Maßnahme im Rahmen des Art. 35 GG zu begründen.

bb) Die Voraussetzungen des Art. 87a Abs. 3 oder Abs. 4 GG liegen offenkundig nicht vor. Ich gehe davon aus, daß das nicht näher ausgeführt zu werden braucht.

cc) Auch die Voraussetzungen eines Einsatzes der Streitkräfte im Rahmen des Art. 24 GG sind nicht gegeben. Die Maßnahmen zur Abwehr sog. renegade flights sind zwar im Rahmen der NATO abgestimmt worden. Es wurde aber ausdrücklich festgelegt, daß der Einsatz von Waffengewalt gegen ein »renegade« Luftfahrzeug unter NATO Kommando nicht erlaubt ist. Die Entscheidungen über Identifizierung, Warnung oder Waffengewalt werden vielmehr ausschließlich zwischen dem deutschen sog. »NLFZ SiLuRa« in Kalkar und den beteiligten Bundesministerien BMI, BMV und dem BMVBW abgestimmt. Sie stehen also ausschließlich im Bereich der nationalen Zuständigkeit und Hoheitsgewalt.

b) Kein Fall zulässiger „Amtshilfe“ im Sinne des Art. 35 GG

Der Einsatz der Bundeswehr kann auch nicht mit Art. 35 GG gerechtfertigt werden. Voraussetzung ist zunächst entweder eine Naturkatastrophe oder ein besonders schwerer Unglücksfall. Diese Voraussetzung ist auch dann gegeben, wenn ein besonders schwerer Unglücksfall, z.B. durch einen Flugzeugabsturz oder ein Eisenbahnunglück vorsätzlich herbeigeführt wurde.

Streitig ist hingegen, ob eine Hilfeleistung nach Art. 35 GG im Wege der Amtshilfe voraussetzt, daß der Unglücksfall bereits eingetreten ist oder ob die Hilfeleistung im Sinne einer Gefahrenabwehr auch angefordert werden kann, um den Eintritt eines derartigen Unglücksfalles zu verhindern. Das klingt zwar vernünftig. Es ist auch polizeiliche Praxis der Länder, bei erwarteten Großereignissen vorsorglich die Polizeikräfte der anderen Länder und den BGS anzufordern und bereitzustellen. Hier soll es aber nicht darum gehen, die logistischen und personellen Kräfte der Bundeswehr anzufordern, etwa zum Transport von Material oder von Personal zur Verstärkung eines Deiches bei einer drohenden Flut. Hier geht es vielmehr darum, die militärischen Kräfte der Bundeswehr anzufordern, um durch sie eine Leistung zu erhalten, zu der weder die ersuchenden Behörden, noch die ersuchte Behörde rechtlich in der Lage sind. Ein kriegsmäßiger Kampfeinsatz der Bundeswehr im Inland mit militärischen Mitteln ist von Art. 35 GG nicht gedeckt. 
Sowohl Art. 35 Abs. 2 wie Abs. 3 GG erlauben die Hilfe der Streitkräfte für die Polizei unter Verantwortung der jeweiligen Landesregierung und auf der Grundlage des jeweiligen Landespolizeirechts. Das gilt auch für Art. 35 Abs. 3 GG, nach dem die Bundesregierung zwar den beteiligten Landesregierungen die Weisung erteilen kann, Streitkräfte zur Hilfe anzufordern. Aber es bleibt bei einer Hilfe im Rahmen polizeilicher Aufgaben und des Katastrophenschutzes unter der Verantwortung der jeweiligen Landesregierung, die den Streitkräften die von ihnen zu bewältigenden Aufgaben zuweist. Dabei scheidet die vorsätzliche Tötung von Personen, die polizeirechtlich als Unbeteiligte gelten, grundsätzlich und ohne Ausnahme nach den Polizeigesetzen aller Bundesländer aus. Sie ist in keinem Fall zulässig.

Der Gesetzgeber versucht diese Konsequenz dadurch zu umgehen, daß er zwar den von ihm gewünschten Einsatz der Bundeswehr in $\S 13$ Abs. 1 LuftSiG als Amtshilfe bezeichnet und die Zuständigkeit des Verteidigungsministers gem. § 13 Abs. 2 Luft$\mathrm{SiG}$ mit seiner Befehlsgewalt in Friedenszeiten begründet (vgl. die Begründung des Gesetzentwurfs a.a.O.), dann aber in $§ 13$ Abs. 4 Satz 2 LuftSiG das Polizeirecht der Länder bei den angeforderten Zwangsmaßnahmen generell durch die Vorschriften des LuftSiG ersetzen will. Damit sprengt er aber die Grundsätze der Amtshilfe ebenso wie die verfassungsmäßigen Regeln des Art. 35 GG. ${ }^{13}$

Die Verfassungsmäßigkeit des Einsatzes der Streitkräfte mit militärischen Mitteln im Inland kann demnach nicht mit Art. 35 GG begründet werden. Sie hängt vielmehr davon ab, ob die $\S \S 13$ ff. LuftSiG mit Art. 87a GG vereinbar sind, in dem sie eine Art partielles Kriegsrecht einführen.

Der Art. 87a GG schließt diese Möglichkeit für den einfachen Gesetzgeber aus. Daß kein Verteidigungsfall vorliegt, wurde schon ausgeführt, vgl. oben Ziff. III 3a. Auch der Gesetzgeber selbst geht in seiner amtlichen Begründung zu $\S 13$ LuftSiG davon aus, daß es sich um einen Einsatz in Friedenszeiten handelt.

Während Art. 87a Abs. 3 GG. den militärischen Einsatz der Streitkräfte im Inland im Spannungs- und Verteidigungsfall auch zur Unterstützung der Polizei regelt, setzt Art. 87a Abs. 4 GG für den militärischen Einsatz der Bundeswehr im Innern den Staatsnotstand voraus. Er begrenzt den Einsatz auf den Fall, daß weder die Polizeikräfte eines Landes noch der BGS ausreichen, um militärisch bewaffnete organisierte Aufständische zu überwinden. Selbst in diesem äußersten Fall sollen Bundestag und Bundesrat berechtigt sein, den Einsatz der Bundeswehr zu beenden. Diese ausdrücklichen Begrenzungen der Verwendung militärischer Mittel im Inland wären weitgehend gegenstandslos, wenn schon die vermutete Absicht, einen schweren Unglücksfall herbeizuführen, die Polizeien berechtigen würde, den militärischen Ein-

13 Die ausschließliche Geltung des Landesrechts beim Einsatz der Streitkräfte wird gelegentlich mit dem Argument bestritten, daß dem Bund die alleinige Gesetzgebungszuständigkeit im Bereich der Luftsicherung zustehe, vgl. Baldus, Streitkräfteeinsatz zur Gefahrenabwehr im Luftraum, NVwZ 2004, 1278 ff. (1284). Dann könnte es sich aber nicht um Amtshilfe für die Landespolizei i. S. d. Art. 35 GG handeln und es würde sich nichts daran ändern, daß der einfache Bundesgesetzgeber bei der Ausgestaltung der Eingriffsrechte der Streitkräfte an die Grenzen des Art. 87a GG gebunden ist, vgl. dazu auch Epping, Anhörungsgutachten, ADrS. 15 (4) 102 B, S. 9. 
satz der Streitkräfte als polizeiliche Amtshilfe anzufordern, sei es mit Kampfflugzeugen, Boden-Luft-Raketen oder Flugabwehrgeschützen. Die verfassungsrechtliche Sperre des Art. 87a GG kann eben nicht durch ein einfaches Gesetz, hier die $\S \S 13,14$, 15 LuftSiG, überwunden werden. ${ }^{14}$

c) Einsatz der Bundeswehr im Inland nur nach ausdrücklicher Verfassungsänderung

Man kann durchaus die Meinung vertreten, daß der Verfassungsgesetzgeber von 1949 oder bei der Einführung des Art. 87a GG 1956 und 1968 die hier behandelte Gefahrenlage nicht kennen konnte. Aber der Verfassungsgesetzgeber war sich durchaus der Versuchungen bewußt, die Bundeswehr, wenn sie erst einmal geschaffen ist, für immer weitere Aufgaben einzusetzen. Dabei muß es nicht einmal um innenpolitischen Machtmißbrauch gehen, sondern um den Gedanken, personal- und kostenintensive Aufgaben bei polizeilichen Großlagen - wie etwa bei Gorleben oder Wackersdorf möglichst auf den Zentralstaat abzuwälzen, wie man das gerade bei der ständigen Aufgabenvermehrung des BGS beobachten kann. ${ }^{15}$

Die Verfassung stellt sicher, daß hinsichtlich der Bundeswehr keine schleichende Verfassungsänderung eintritt und eine Ausdehnung der militärischen Aufgaben der Bundeswehr nur durch eine ausdrückliche Verfassungsänderung erfolgen darf. Eine solche Verfassungsänderung ist nicht erfolgt, weil man sich darüber nicht einigen konnte. Sie kann auch nicht ersatzweise durch eine bloße ausdehnende Interpretation des Art. 35 GG herbeigedacht werden (vgl. dazu oben Zif. III 3 a und d. d. Zit).

\section{Ergebnis}

(1) Die mit der Verfassungsbeschwerde angefochtenen $\S \S 13,14$ und 15 LuftSiG sind verfassungswidrig. Sie verletzten die Grundrechte der Beschwerdeführer aus Art. 1 und 2 GG, ihre Grundrechte auf Menschenwürde und Leben. Sie verletzten die Grundrechte der Beschwerdeführer auch dadurch, daß sie den Bundesverteidigungsminister ermächtigen, sie unter bestimmten Umständen durch einen verfassungswidrigen Einsatz der Bundeswehr töten zu lassen.

14 Vgl. dazu auch mit umfangreicher Literatur: Dreist, »Terrorismusbekämpfung als Streitkräfteauftrag - Zu den verfassungsrechtlichen Grenzen polizeilichen Handelns der Bundeswehr im Innern«, Der Kriminalist, 2003, 349 ff. u. ders., »Offene Rechtsfragen des Einsatzes bewaffneter deutscher Streitkräfte«, NZWehrr 2002, 133 ff. Ferner: Wilkesmann, »Terroristische Angriffe auf die Sicherheit des Luftverkehrs«, NVwZ 2002, 1316 ff., der ebenfalls alle hier erörterten Eingriffsgrundlagen sowie ein staatliches Notstandsrecht nach § 34 StGB wegen der Tötung Unschuldiger ablehnt, dann aber den Abschuß eines von terroristischen Selbstmordattentätern entführten Flugzeugs ohne weitere Begründung akzeptiert. Auch Lutze »Abwehr terroristischer Angriffe als Verteidigungsaufgabe der Bundeswehr«, NZWehrr 2003, 101 ff., lehnt alle hier erörterten Eingriffsgrundlagen ab, hält aber einen Verteidigungsfall dann für gegeben, wenn es sich um einen Anschlag einer terroristischen Organisation handelt, wenn sie von außen gesteuert wird und eine militärähnliche Struktur aufweist. Unklar bleibt, wie man bei einem renegade flight von außen erkennen soll, ob diese Voraussetzungen vorliegen.

15 Vgl. so im Ergebnis auch Schmidt-Jortzig, »Verfassungsänderung für Bundeswehreinsatz im Innern Deutschlands?«, DÖV 2002, 773 ff. 
Der militärische Einsatz der Bundeswehr im Inland gegen terroristische oder andere kriminelle Straftaten oder gegen Gefährdungen durch Irre wird von der Verfassung nicht erlaubt.

Die vorsätzliche Tötung unschuldiger Menschen, die selbst Opfer eines Verbrechens sind und von denen keine Gefahr ausgeht, kann auch durch eine Verfassungsänderung nicht erlaubt werden. Wenn uns Terroristen dazu zwingen könnten, einen solchen elementaren Rechtsgrundsatz aufzugeben, dann hätten sie wirkliche Macht, auch über unsere Gedanken, errungen. Wenn der Staat demjenigen, von dem »den Umständen nach davon auszugehen ist «, daß er bald sterben muß, das Recht auf Leben entziehen könnte, dann würden wir aufhören, ein Rechtsstaat zu sein.

(2) Die Bundesregierung wird also andere Überlegungen anstellen müssen, wie sie der Bedrohung vorbeugend begegnen kann, wenn sie die Gefahr für konkret hält. Sie darf mit ihren Überlegungen nicht erst dann einsetzen, wenn es für verfassungsmäßiges Handeln schon zu spät ist. Das mag lästig und unangenehm sein, weil solche Maßnahmen Zeit und Geld kosten, so z. B. verstärkte Kontrollen i. S. der EG-VO 2320/2002 v. 16. Dez. 02 (Amtsbl. L 355/1 ff ), individuelle Gepäckidentifizierungen, der regelmäßige Einsatz bewaffneter Flugbegleiter oder bestimmte neue technische Ausrüstungen der Flugzeuge. Aber sie sind jedenfalls mit der Verfassung vereinbar.

(3) Wir sind der Überzeugung, daß der Gesetzgeber kein Mitglied der Bundesregierung, mag es der Verteidigungsminister oder der Bundeskanzler sein, in die Lage bringen sollte und bringen darf, nach seinem Ermessen die vorsätzliche Tötung unschuldiger Menschen von Rechts wegen anordnen zu können. Ein Gesetz nimmt ihm weder die rechtliche, noch die politische oder gar moralische Verantwortung für die Frage $\mathrm{ab}$, wie er sich entscheiden soll. Ob das Gesetz es erlaubt und ob es dabei auch einen tatsächlichen Irrtum deckt oder nicht, er verantwortet das Ergebnis selbst und niemand sonst. Und er wird niemals wissen, ob er mit seiner Entscheidung tatsächlich Menschen gerettet oder ob er nur getötet hat. Er mag in bester Absicht gehandelt haben, aber er bleibt ein Täter.

(4) Das gilt auch für den Piloten der Bundeswehr, der sich darauf berufen könnte, »auf Befehl« gehandelt zu haben, ohne selbst beurteilen zu können, ob der Befehl »richtig « war oder nicht. Der Pilot hat es nicht mit Kombattanten in einem Krieg zu tun oder mit gefährlichen Straftätern, sondern mit ganz normalen Menschen. Er tötet sie auf eine technisierte Weise. Er wird nicht zum Helden, sondern zur Waffe. Ob er dabei auf Befehl handelt oder nicht - er ist es, der sie tötet, ohne jemals wissen zu können, ob er dadurch andere Menschen gerettet oder ob er nur getötet hat. Er mag in bester Absicht gehandelt oder sich subjektiv zum Werkzeug fremder Entscheidungen erniedrigt haben, er bleibt ein Täter.

Dieses Gesetz erweckt nur den Anschein, eine Lösung zu sein und »Handlungsfähigkeit« zu beweisen. Aber in Wirklichkeit löst es kein Problem und hilft niemandem. Es verführt zu dem Irrtum, einem Gesetz zu gehorchen, wo man nur dem eigenen Gewissen gehorchen kann.

(5) Wir begrüßen es, daß der Bundespräsident in seiner Presseerklärung v. 12. Jan. 2005 anläßlich der Unterzeichnung des Gesetzentwurfes seine Zweifel an der Verfassungsmäßigkeit des LuftSiG zum Ausdruck gebracht hat. Wir sehen aber davon ab, 
den Erlaß einer einstweiligen Anordnung zu beantragen. Der Vorgang ist zu gravierend, als daß er in einem Eilverfahren vorentschieden werden sollte.

Wir vertrauen darauf, daß sich das Beschwerdeverfahren nicht lange hinziehen wird und erwarten von der Bundesregierung, daß sie die Piloten der Bundeswehr nicht in die Lage bringen wird, Menschen auf der Grundlage eines Gesetzes erschießen zu sollen, dessen verfassungsrechtliche Nachprüfung selbst vom Bundespräsidenten nahegelegt worden ist. 